\title{
Microstructured surface design for omnidirectional antireflection coatings on solar cells
}

\author{
Weidong Zhou, ${ }^{\text {a) }}$ Meng Tao, ${ }^{\text {b) }}$ Li Chen, and Hongjun Yang \\ Department of Electrical Engineering, University of Texas at Arlington, Arlington, Texas 76019-0072, USA
}

(Received 4 August 2007; accepted 26 September 2007; published online 27 November 2007)

\begin{abstract}
We recently demonstrated a new process for the formation of partially spherical structures as an omnidirectional antireflection coating (omni-AR). In this paper, we report the simulation results of the angular and spectral dependences of the total reflectivity on various microstructured surfaces based on the rigorous coupled-wave analysis. Close to zero reflection can be achieved in these microstructured surfaces over an extended spectral region for large ranges of light incident angles. The impact of feature size, density, shape, and refractive index has all been investigated. The experimental results agree reasonably well with the theoretical work. Such an omni-AR structure offers an attractive solution to current crystalline silicon solar cells, as well as future thin film, quantum dot, and organic solar cells. (C) 2007 American Institute of Physics.
\end{abstract}

[DOI: $10.1063 / 1.2817470]$

\section{INTRODUCTION}

A flexible optical design for light collection is vital in achieving high performance solar cells. ${ }^{1}$ An ideal antireflection (AR) structure should lead to zero reflection loss on solar cell surfaces over an extended solar spectral range for all angles of incidence. Such a coating can eliminate the need for a mechanical tracking device for proper optical alignment of the solar cell with respect to incident sunlight. ${ }^{1}$ A perfect omnidirectional AR (omni-AR) structure has been a subject of intensive research in thin film optics and most importantly, for solar cell applications. A series of papers were published by Dobrowolski and Poitras et al. in search for the perfect AR structure. ${ }^{2-4}$

In theory, there are three major types of nonabsorbing omni-AR structures. The first group is the single or multilayer quarter-wavelength film stacks. ${ }^{5,6}$ Close to zero reflection over a certain spectral range for wide ranges of incident angles can be achieved by controlling the refractive index and thickness of each individual layer in the multilayer structure. The major challenges for this approach are the availability of materials with the right indexes and good optical transparency, as well as the precise control of film thickness. In solar cell applications, a single layer thin film AR coating, e.g., silicon nitride $\left(\mathrm{SiN}_{x}\right)$ thin film for silicon $(\mathrm{Si})$ solar cells, is often used as a cost effective approach. Such a single layer AR coating reduces reflectivity only in a limited spectral range under surface normal incident conditions.

The second group is the graded index (GRIN) AR structures, ${ }^{7}$ where the refractive index of the structure changes gradually from the top to the bottom. ${ }^{8,9}$ The index profile can follow different mathematical functions, such as linear, cubic, or quintic. With a GRIN AR structure, extremely low reflection can be achieved over a broad spectral range for a wide range of incident angles, especially with the quintic index profile. ${ }^{7}$ The difficulty associated with the

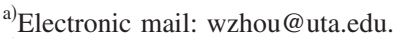

b)Electronic mail: mtao@uta.edu.
}

choice of materials for practical control of index profiles has prevented this structure from practical applications. Sol-gel based approaches with either porosity control and/or high index dopant introduction were reported. ${ }^{10-12}$ Recently a $\mathrm{SiO}_{2} / \mathrm{TiO}_{2}$ GRIN AR coating was reported by $\mathrm{Xi}$ et al., ${ }^{13}$ by oblique-angle physical vapor deposition, where the refractive index can vary from 1.0 to 2.0 .

The third group is the textured surface AR structures. The most successful example is the anisotropic etching of single crystal $\mathrm{Si}(100)$ surface in a solution containing potassium hydroxide. ${ }^{9,14-17}$ It has led to record solar cell efficiencies in the laboratory and has been the standard AR structure on all commercial single crystal Si solar cells. ${ }^{9,14-17}$ Close to zero reflection over a wide spectral range can be achieved when an additional layer of low index film (e.g., $\mathrm{SiN}_{x}$ ) is coated on these microscale pyramidal structures on the $\mathrm{Si}$ surface. However, the microscale surface texture involves anisotropic etching of the Si substrate, which does not apply to polycrystal $\mathrm{Si}$ and non-Si solar cells. In this case, anisotropic etching becomes too unreliable and photolithographydefined surface textures too expensive.

In this paper, we will introduce a new process for microscale surface texturing for omni-AR coatings. ${ }^{18,19}$ The process is solution based, which is compatible with large scale manufacturing and flexible for integration on various solar cell substrates. It is inherently low-cost and energy efficient, without complicated large vacuum systems. A detailed theoretical investigation has been carried out to understand the optical performance of the microscale surface textures for AR applications. We compared hemispherical structures to pyramidal and conical structures. The impact of refractive index, feature size, and density is also reported. Finally, experimental results from omni-AR coatings on quartz substrates are compared with simulation results.

\section{OMNI-AR COATINGS FROM SPHERICAL PARTICLES}

The basic structure of the omni-AR coating is shown in Fig. 1(a). The coating comprises an array of, in an ideal 
(i) Monolayer self assembly of spherical particles

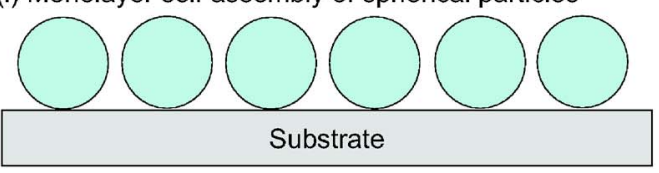

(ii) Spin coating of sol-gel or SOG glass based thin films

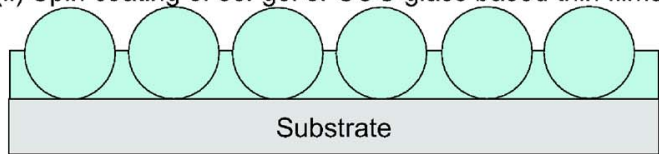

(iii) Formation of omni-directional anti-reflection coating

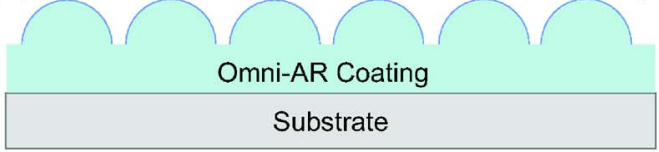

(a)

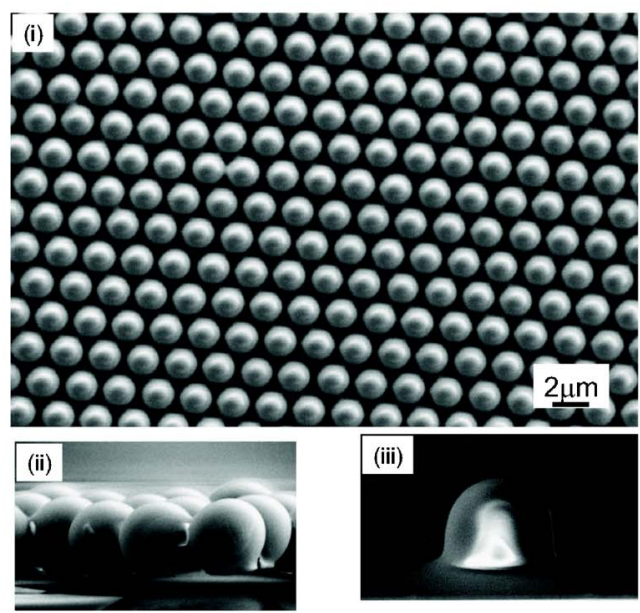

(b)

FIG. 1. (Color online) (a) Omni-AR coating based on monolayer of spherical particles and SOG film. (b) Scanning electron micrographs of fabricated omni-AR coatings: (i) top view; (ii) cross-sectional view before SOG film, and (iii) cross-sectional view after SOG film.

example, hemispherical and microscale dielectric particles. The hemispherical particles are formed by partially immersing microscale spherical particles into a dielectric film of the same refractive index. Both the particle array and the dielectric film can be prepared from solutions containing microscale particles and precursors for the dielectric film. The performance of the coating can be controlled by tuning the size and packing density of the dielectric particles and the thickness and refractive index of the dielectric film, as discussed in this paper. Shown in Fig. 1(b) are scanning electron micrographs of processed omni-AR structures on quartz substrates. A closely packed monolayer of $2-\mu \mathrm{m}$ silica spheres were first deposited on the substrate, followed by coating of a spin-on-glass (SOG) film with a desired thickness, with Fig. 1(b) sowing a $0.2-\mu \mathrm{m}$ SOG film. Finally, an omni-AR structure is formed after baking to cure the SOG film. Detailed experimental conditions can be found in Ref. 19.

\section{DESIGN AND SIMULATION}

\section{A. Simulation approach}

The geometrical structure under consideration is depicted in Fig. 2. A square lattice of hemispheres is con-

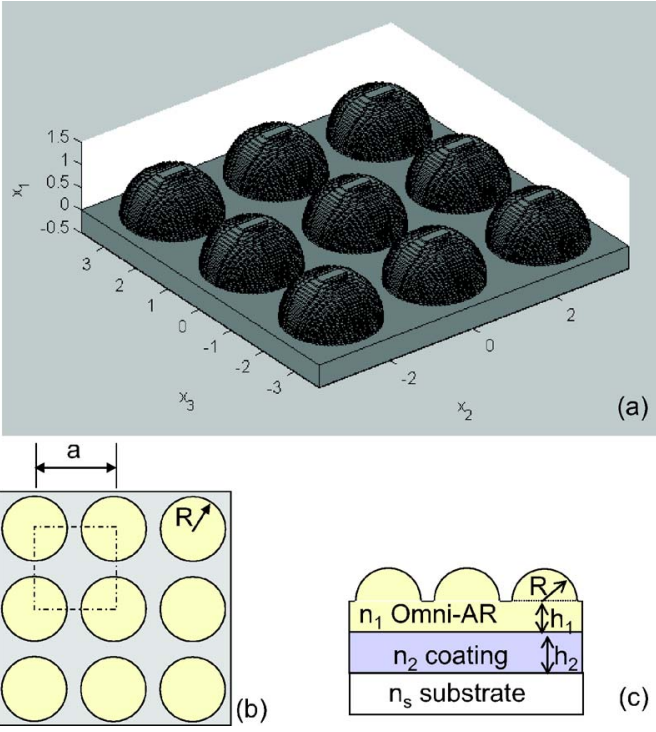

FIG. 2. (Color online) A hemispherical grating as the basic anti-reflection structure for simulation: (a) three-dimensional view of the square lattice; (b) top view, and (c) cross-sectional view of the basic structure.

structed on an optional dielectric film, which sits on a substrate. The hemispherical omni-AR structure, optional dielectric film, and substrate may have the same or different refractive indices $n_{1}, n_{2}$, and $n_{s}$, respectively. The structural parameters, as shown in the top view and cross-sectional view of the omni-AR structure in Fig. 2, are variables in our simulations to investigate their impact on reflectivity.

The substrate material is $\mathrm{Si}$ in most cases, though the structure proposed here can be applied to other substrate materials with different optical properties. In some cases quartz substrates are used in order to be able to measure the total transmissivity over the visible spectral region. Shown in Fig. 3 (a) is the dispersion curves for the refractive index and extinction coefficient for $\mathrm{Si}$ used in our simulations. ${ }^{20}$ The complex index is expressed as $\boldsymbol{n}=n+i k$, where $n$ and $k$ are the real and imaginary part of the refractive index, respectively. The imaginary part $k$, i.e., the extinction coefficient, is related to the absorption coefficient $\alpha$ with $\alpha=4 \pi k / \lambda$, where $\lambda$ is the free-space wavelength. Note the refractive index of $\mathrm{Si}$ changes with the wavelength over the spectral region of interest. For practical designs, the impact of absorption from the Si substrate should be considered, especially when the $k$ value is comparable to the $n$ value, e.g., in the ultraviolet region [for photon energy greater than $3 \mathrm{eV}$ or wavelength below $400 \mathrm{~nm}$, as shown in Fig. 3(a)]. We did not consider absorption in our simulation, as it will not alter significantly the reflectivity results in our simulation. This is because in the wavelength region of $400-1600 \mathrm{~nm}$, the extinction coefficient for Si substrate is relatively small $(k<0.4)$ as compared to the real part of the index $(n>3.5)$. The primary focus of our work is on light propagation through an omni-AR structure on a $\mathrm{Si}$ substrate, where the omni-AR structure has no or little absorption. The refractive index dispersion curves for $\mathrm{Si}$ and silicon dioxide $\left(\mathrm{SiO}_{2}\right)$ used in our simulation in the wavelength region of interest is shown in Fig. 3(b). 


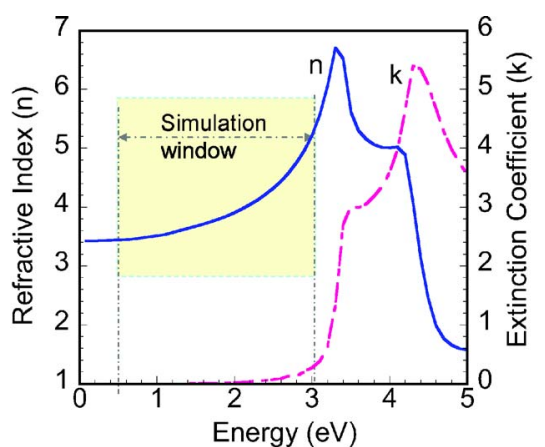

(a)

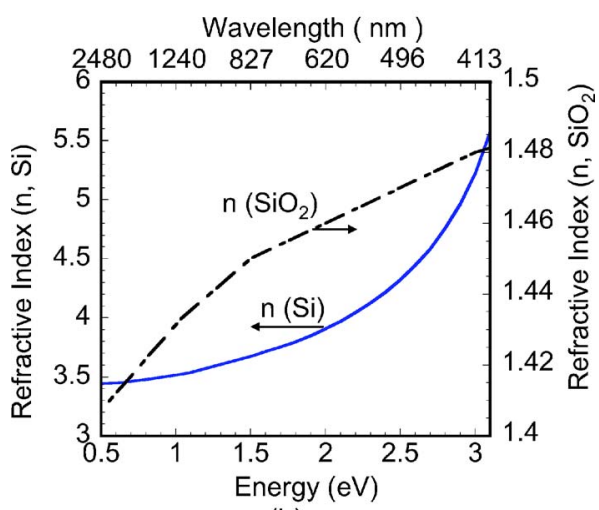

(b)

FIG. 3. (Color online) (a) Crystal silicon complex index dispersion curve $\boldsymbol{n}=n+i k$ from ultraviolet to infrared and (b) refractive index dispersion curve of silicon and silicon dioxide used in our simulation.

The simulation is based on rigorous coupled-wave analysis, ${ }^{5,21,22}$ which analyzes the diffraction of an electromagnetic plane wave incident obliquely on a lossless grating structure composed of materials with different indices. The fields inside all the layers are expanded in a sum of spatial harmonics, i.e., Fourier expansion. This expansion can be expressed and solved as an eigenvalue problem for all of the harmonic orders. For each order of the field components, the wave undergoes simultaneously forward and backward diffraction in a predefined periodic structure with different indices. By solving the coupled wave equations for both transmitted and reflected waves, the fundamental and higher order forward and backward wave diffraction efficiencies are determined. The final solution to the electric field will be the sum of a series of Fourier components based on the solutions from the earlier coupled wave equations. Both total transmission and reflection waves can finally be computed.

All the simulations done here include both transverseelectric (TE) and transverse-magnetic (TM) polarizations. Since we are mostly concerned with sunlight, which is nonpolarized, all the reflectivity results are a simple average of reflectivities calculated from both TE and TM polarized incident waves. Note the calculation counts for both the specular (zero order) component as well as the diffusive (higher order) component as the total reflected/transmitted power. This also agrees well with our experimental setup, where an integrating sphere is used for total reflection/transmission measurement.

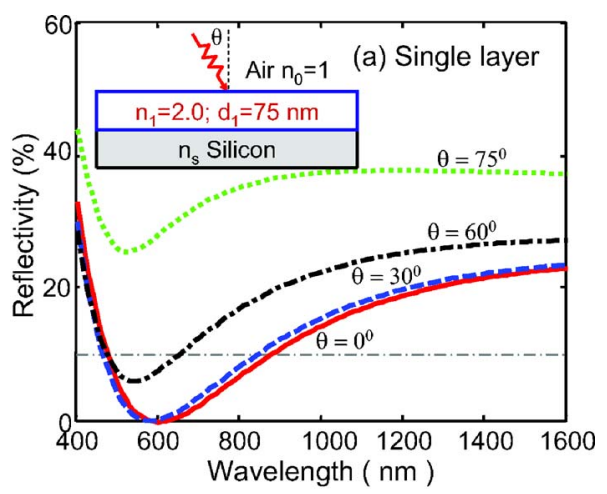

(a)

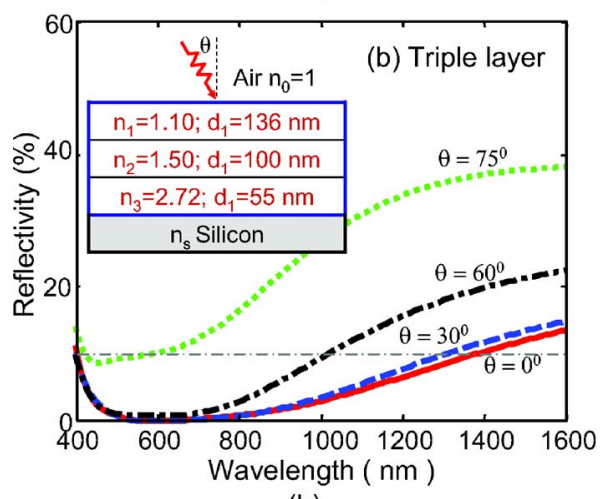

(b)

FIG. 4. (Color online) Simulated quarter wavelength multilayer thin film AR structures with ideal indices and thicknesses for (a) single and (b) triple layers.

\section{B. Multilayer thin film structures}

For comparison, we first considered quarter wavelength multilayer thin film AR structures. Assuming ideal indices for all the layers and with optical thicknesses targeting 600 $\mathrm{nm}$ wavelength, we simulated different AR structures of single and triple layers, as shown in Fig. 4. The ideal indices are chosen to achieve close to zero reflection at the target wavelength (600 $\mathrm{nm}$ in this case). For single layer AR coating (ARC), the ideal index for the coating layer $n_{1}$ follows the equation of $n_{1}^{2}=n_{0} n_{s}$, where $n_{0}$ and $n_{s}$ are the indices of top (air) and bottom ( $\mathrm{Si}$ ) layers, respectively [inset of Fig. 4(a)]. Similarly, the ideal indices for double layer and triple layer ARC are $\left(n_{2} / n_{1}\right)^{2}=n_{s} / n_{0}$ and $\left(n_{1} n_{3} / n_{2}\right)^{2}=n_{0} n_{s}$, respectively [inset of Fig. 4(b)]. As expected, for the single layer AR structure, close to zero reflectivity can be achieved only at the target wavelength with normal incident direction. By increasing the number of layers, it is possible to achieve relatively wider spectral coverage with close to zero reflection in a reasonably wide range of incident angles (up to $\left.60^{\circ}\right)$.

\section{Hemispherical surface and omni-AR structures}

We then considered hemispherical structures with different structural parameters. In what follows, we assume the substrate material is $\mathrm{Si}$, and the coating material is $\mathrm{SiO}_{2}$, with a refractive index dispersion curve shown in Fig. 3(b) $\left(n_{\mathrm{SiO}_{2}} \sim 1.45\right)$. Starting with a simple case in Fig. 5(a), where a hemispherical structure is coated on a Si substrate, with the 


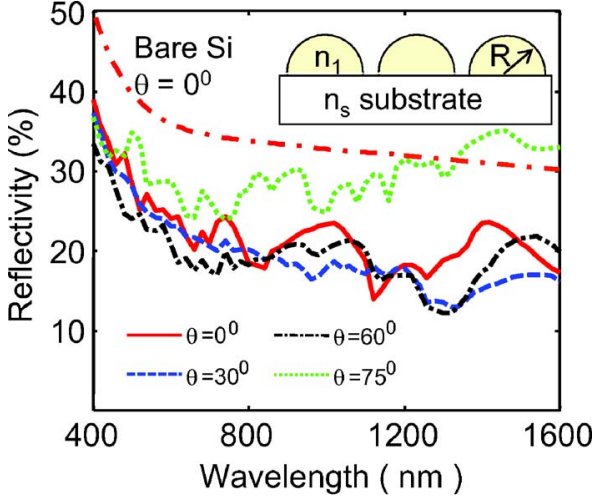

(a)

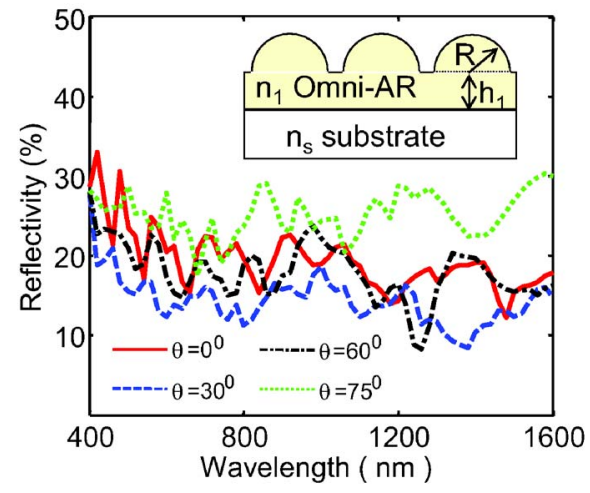

(b)

FIG. 5. (Color online) Simulation results for hemispherical structures with index $n_{1}=1.5$ on silicon substrate: (a) square lattice hemispherical structure with radius $R=1 \mu \mathrm{m}$ and lattice constant $a=2.5 \mu \mathrm{m}$ and (b) omni-AR structure with hemispherical structure on top of a thin film with the same index $n_{1}$ and thickness $h_{1}=R=1 \mu \mathrm{m}$.

hemispherical radius $R=1 \mu \mathrm{m}$ and lattice spacing $a$ $=2.5 \mu \mathrm{m}$. The reflectivity is reduced by $\sim 50 \%$ for incident angles up to $60^{\circ}$ over the entire spectral region of simulation, as compared to the reflectivity for a bare Si substrate at surface normal incident conditions. The reflectivity for incident angle of $75^{\circ}$ is also reduced in the shorter wavelength regime. The result is encouraging, as it proves the incident angle independence, thus omnidirectional, of the hemispherical structure we investigated here.

It is worth pointing out that the hemispherical structure we considered here is not ideally closely packed, which could lead to underestimation in performance as compared to a close-packed structure (with $a=2 R$ ), especially at surface normal or small incident angles. The impact of packing density will be discussed later. The loosely packed structure in our simulation is a better representation of the actual experimental conditions, where silica particles are not closely packed together to form a monolayer over a large area.

The low index film between substrate and hemispherical structure, as shown in Fig. 5(b), is inherently present in the structure we proposed in Sec. II. We investigated the impact of such a thin film $\left(h_{1}\right)$ on reflectivity, as shown in Fig. 5(b), for different incident angles. Compared to Fig. 5(a), we see insignificant changes in reflectivity. The oscillations in Fig. 5 are most likely due to interference, which is to be investigated.

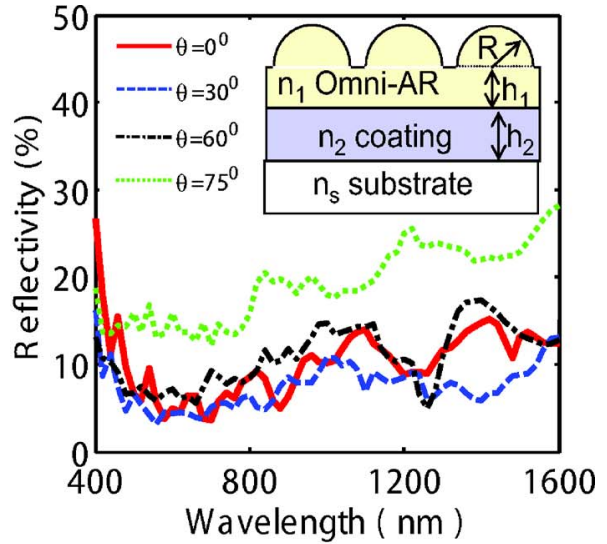

(a)

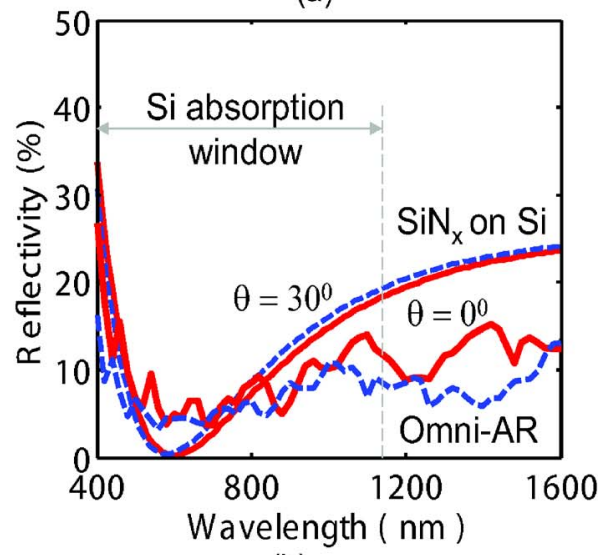

(b)

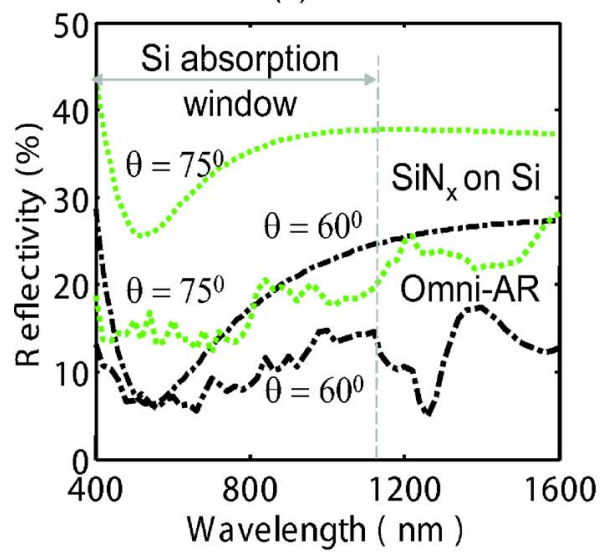

(c)

FIG. 6. (Color online) (a) Simulation results for omni-AR structure on $\mathrm{Si}$ substrate with a $\operatorname{SiN}_{x}$ thin film in between $\left(n_{2}=2.0\right.$ and $\left.h_{2}=75 \mathrm{~nm}\right)$; other simulation parameters are the same as those in Fig. 5(b). The reflectivity for (b) small incident angles $\left(\theta=0^{\circ}\right.$ and $\left.30^{\circ}\right)$ and (c) large incident angles $(\theta$ $=60^{\circ}$ and $75^{\circ}$ ) for two cases: $\mathrm{SiN}_{x}$ coated $\mathrm{Si}$ and omni-AR on $\mathrm{SiN}_{x}$ coated Si.

We further considered another scenario where the omni-AR structure is an add-on to current polycrystal Si solar cells, where a single layer of $\operatorname{SiN}_{x}$ is coated on top of the solar cells. The results are shown in Fig. 6(a). Significantly reduced reflectivity can be achieved over a wide spectral range for incident angles from $0^{\circ}$ to $60^{\circ}$. A comparison is given in Figs. 6(b) and 6(c) for $\mathrm{SiN}_{x}$ coated $\mathrm{Si}$ substrates with and without omni-AR coatings. We see the improvement in reflectivity is relatively small for small incident angles of $0^{\circ}$ and $30^{\circ}$ [Fig. 6(b)]. However, significant im- 


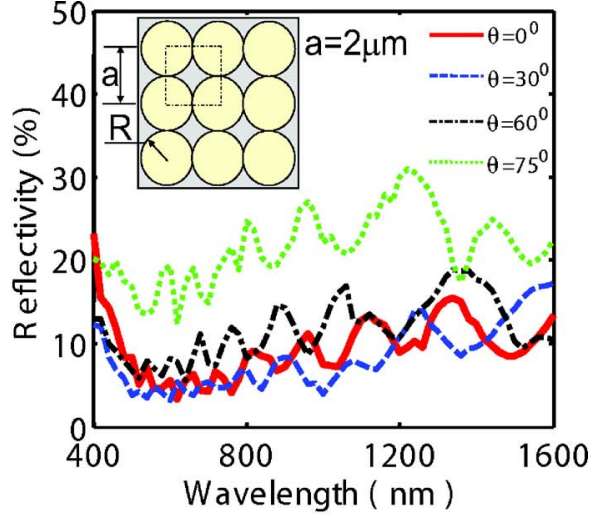

(a)

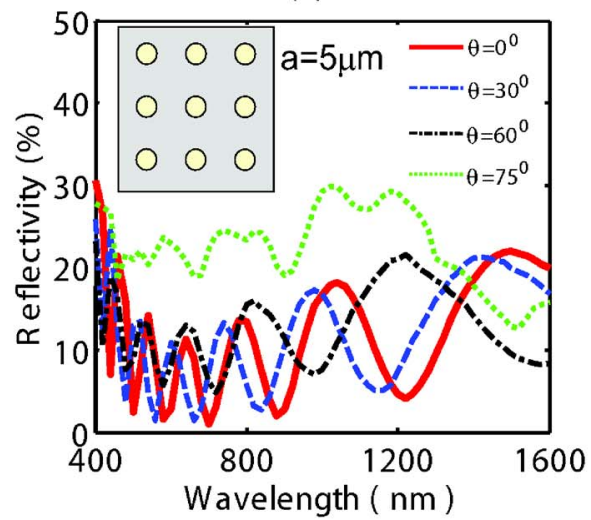

(b)

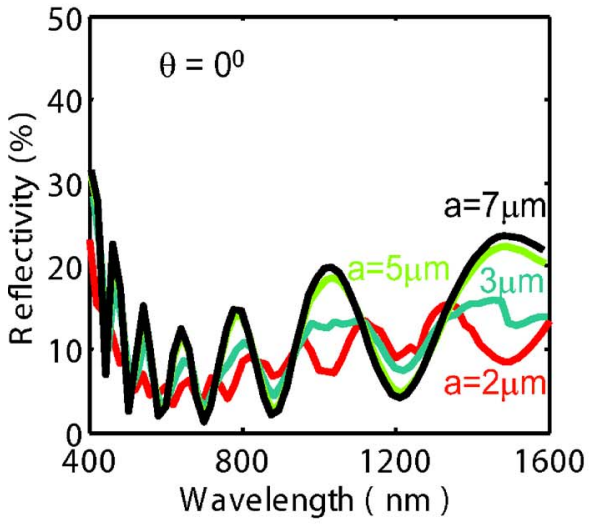

(c)

FIG. 7. (Color online) Packing density impact for the proposed hemispherical omni-AR structure similar to the one in Fig. 6(a) with $R=1 \mu \mathrm{m}, n_{1}$ $=1.5$, and lattice constant (a) $a=2 \mu \mathrm{m}$ and (b) $a=5 \mu \mathrm{m}$. (c) Surface normal reflectivity for different packing densities.

provement in reflectivity can be achieved for large incident angles of $60^{\circ}$ and $75^{\circ}$ [Fig. 6(c)]. This is the most attractive feature of the omni-AR structure, which provides angle independent reflectivity over a wide spectral range.

The impact of packing density and particle size was investigated as well. Shown in Fig. 7 is the simulated reflectivity with different packing densities. With fixed radius $R$ $=1 \mu \mathrm{m}$, the packing density can be varied by changing the lattice constant $a$. Shown in Figs. 7(a) and 7(b) are two examples with $a=2 \mu \mathrm{m}$ and $a=5 \mu \mathrm{m}$, respectively. The surface normal reflectivity for different packing densities is shown in Fig. 7(c). It is worth noting that the simulated reflectivity does not change significantly over a relatively

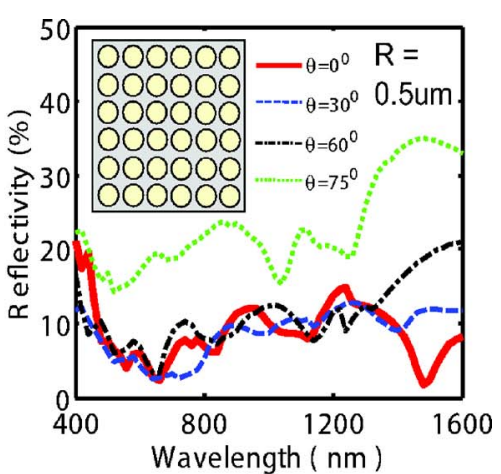

(a)

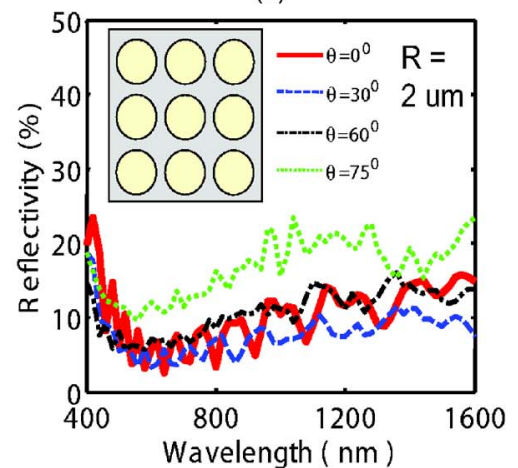

(b)

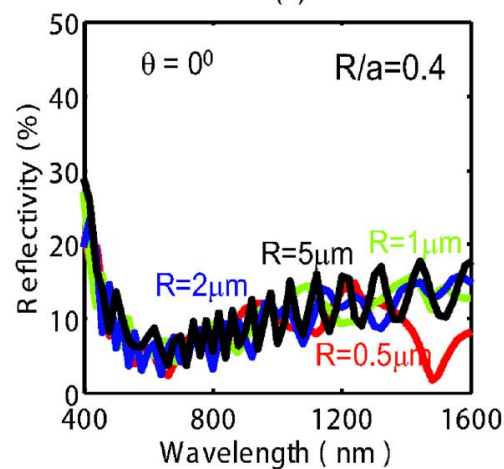

(c)

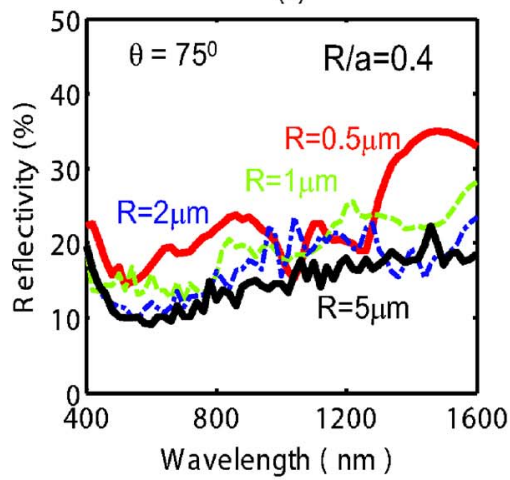

(d)

FIG. 8. (Color online) Spherical radius impact for the proposed hemispherical omni-AR structure similar to the one in Fig. 6(a) with $R / a=0.4, n_{1}$ $=1.5$, and spherical radius (a) $R=0.5 \mu \mathrm{m}$ and (b) $R=2 \mu \mathrm{m}$. (c) Surface normal reflectivity and (d) large incident angle reflectivity for different spherical radius.

large range of packing densities. The only notable feature is the increased oscillation for smaller packing densities. This could be due to mainly interference in the layer under the particles $\left(h_{1}\right)$ layer in the inset of Fig. 6(a), as the flat surface area increases with the reduction of packing density. 
(a) Pyramid-Shaped

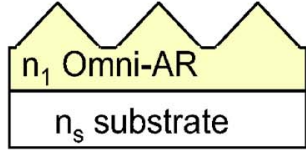

Loose-packed Structure
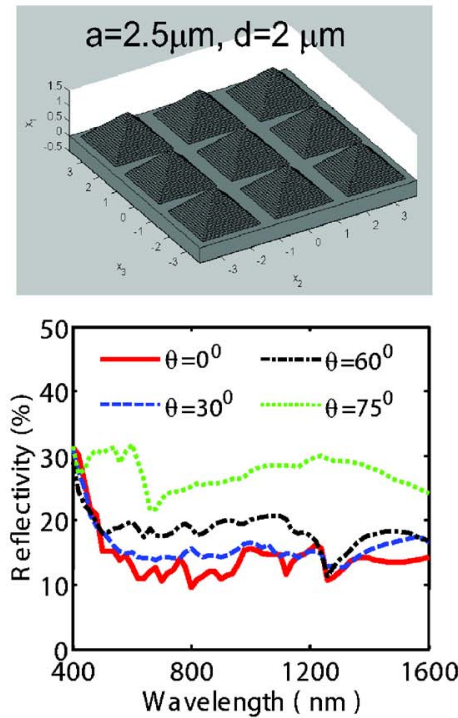

Close-packed Structure
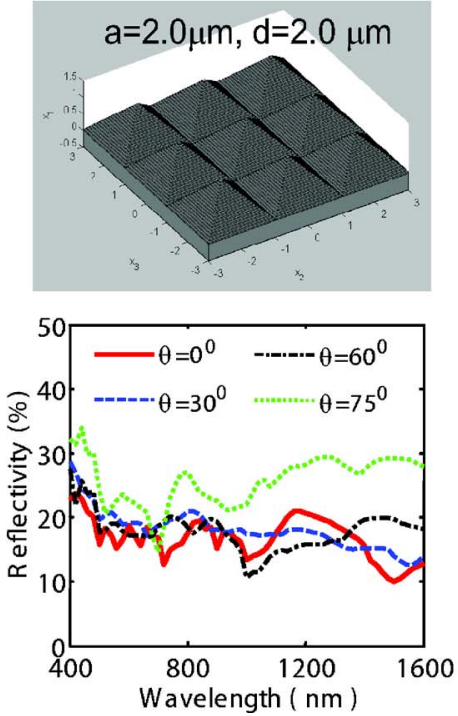

(b) Cone-Shaped
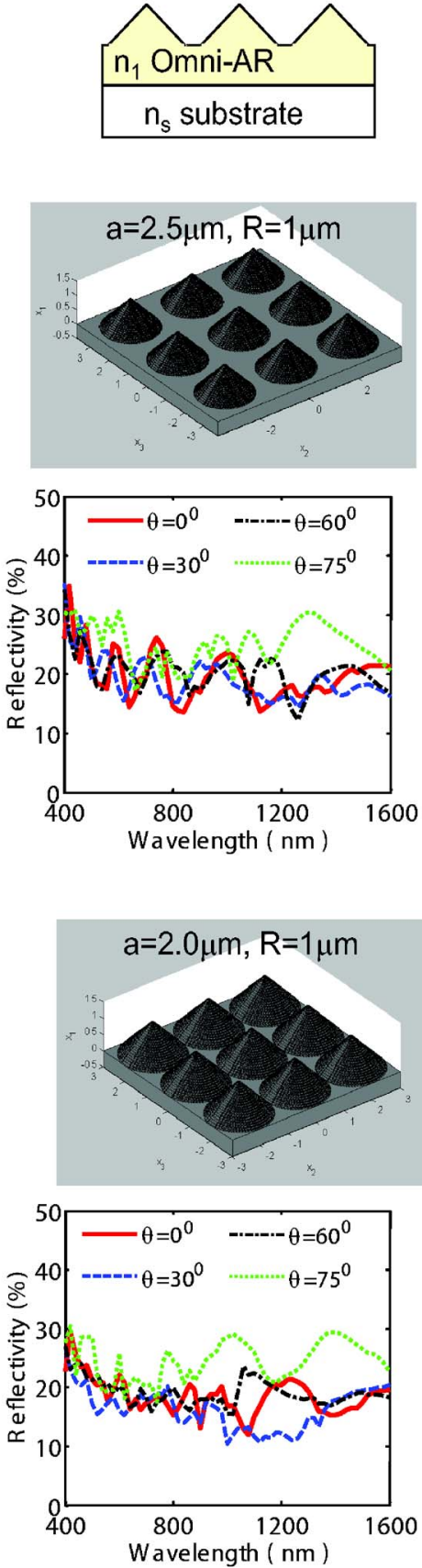

(c) Hemisphere-Shaped
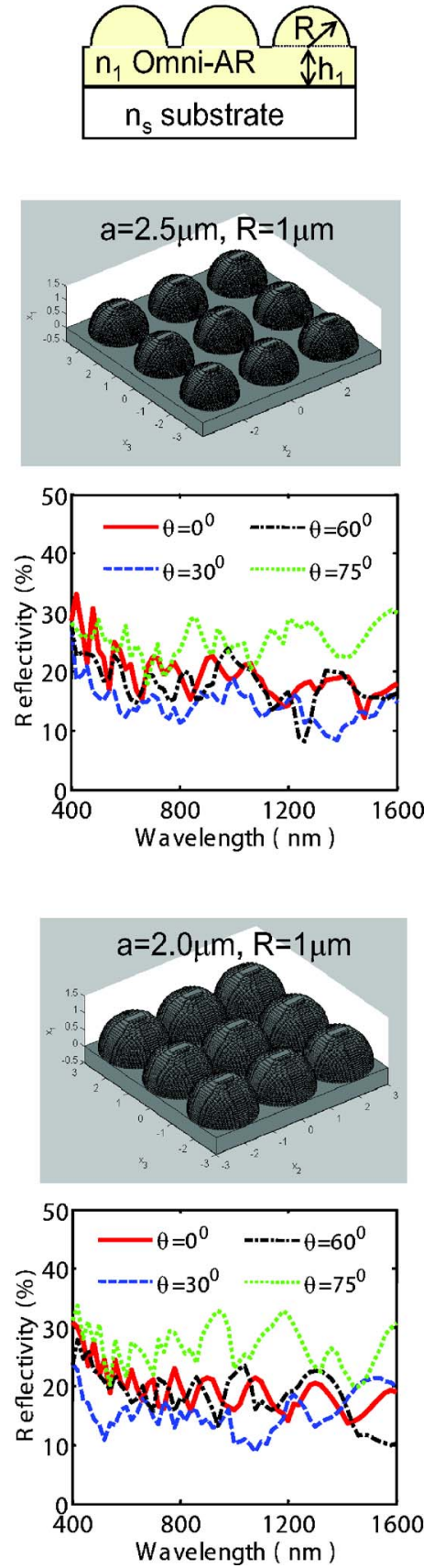

FIG. 9. (Color online) The structures and simulation results for three differently shaped structures: (a) pyramids, (b) cones, and (c) hemispheres. Both loosely packed and close-packed structures are simulated for comparison.

Figure 8 is the simulated reflectivity for different particle sizes. Here the packing fraction is fixed, i.e., the ratio between radius $R$ and lattice constant $a$ is fixed at 0.4. Shown in Figs. 8(a) and 8(b) are two examples with $R=0.5 \mu \mathrm{m}$ and $R=2 \mu \mathrm{m}$, respectively. The surface normal reflectivity and large incident angle reflectivity $\left(\theta=75^{\circ}\right)$ for different particle sizes is shown in Figs. 8(c) and 8(d), respectively. Again, we do not see significant differences in the simulated reflectivity, especially at small incident angles up to $60^{\circ}$. These results suggest large process windows in manufacturing the proposed structure for solar cell applications. However, for a larger incident angle $\left(\theta=75^{\circ}\right)$, we see slightly reduced reflectivity with the increase of particle size, which is due to a thicker transition layer at large incident angles. In all the simulations shown in Fig. 8, the thickness of the $h_{1}$ layer (1 $\mu \mathrm{m})$ as shown in the inset of Fig. 6(a) and the packing density (proportional to the ratio of $R / a, 0.4$ ) are kept the same. The fixed and loose packing density leads to open flat areas between hemispheres which increase with the increase of particle size. This may be the cause for the more profound interference features observed for larger particle sizes.

\section{Pyramidal, conical, versus hemispherical structures}

Surface structures of different shapes were also investigated. Shown in Fig. 9 are the structures and simulation re- 
sults for three different shapes: (a) pyramids, (b) cones, and (c) hemispheres. All the structures under simulation have similar structural parameters. The structure material is $\mathrm{SiO}_{2}$ and the substrate is Si. The lattice constant $a=2.5 \mu \mathrm{m}$ and the film thickness $h_{1}=1 \mu \mathrm{m}$. The pyramid base and height are $2 \times 2 \times 1 \mu \mathrm{m}$. The cone base radius is $R=1 \mu \mathrm{m}$ and the height is also $1 \mu \mathrm{m}$. The hemisphere radius is $R=1 \mu \mathrm{m}$. We also simulated structures with close-packed pyramidal particles where the base size equals the lattice constant $a$ $(2 \mu \mathrm{m})$. Based on the simulated results, the hemispherical structure has slightly better performance overall especially at large incident angles, while the pyramidal structure has better performance at small incident angles and at shorter wavelengths. This could be due to the enhanced second strike effect for the pyramidal structure at small incident angles, ${ }^{16}$ as compared to the hemispherical structure. We can also argue that the impact of packing density is very small on the total reflectivity for the three different shapes. This further validates the conclusion based on Fig. 7, which indicates large process windows for the omni-AR structure.

\section{E. Index matching to Si substrate}

The hemispherical structure in the proposed omni-AR offers spectrally and directionally independent reflectivity. Further reduction in reflectivity can be provided with index matching of the omni-AR structure to substrates with different indices. We investigated different GRIN schemes. We first considered the omni-AR structure to be made of $\mathrm{SiO}_{2}$ with index $n_{1} \sim 1.45$. Compared to the reflectivity from a structure with step indices, i.e., $\mathrm{SiO}_{2}$ omni-AR on a $\mathrm{SiN}_{x}$ coated $\mathrm{Si}$ substrate [Fig. 10(a)], the reflectivity is reduced from $\sim 10 \%$ to $\sim 2 \%$ for a structure with graded index $\left(n_{2}\right.$ changes from 1.5 to 4.0 ) between omni-AR and Si substrate for incident angles up to $60^{\circ}$ [Fig. 10(b)].

If the index profile of the omni-AR structure can be adjusted, we observe the effect of index profile on reflectivity. Shown in Fig. 11(a) is a case where the omni-AR index varies from air to $\operatorname{SiN}_{x}\left(n_{1}\right.$ changes from 1.0 to 2.0) with a fixed-index film later. We only see a slight reduction in reflectivity over Fig. 10(a). On the other hand, if the omni-AR index varies from air to the $\mathrm{Si}$ substrate $\left(n_{1}\right.$ changes from 1.0 to 4.0) without the $\mathrm{SiN}_{x}$ film in between, we see close to zero $(<4 \%)$ reflectivity over the entire spectral region of simulation for light with incident angles less than $60^{\circ}$. Such an ideal omni-AR structure offers significantly improved performance.

\section{EXPERIMENTAL RESULTS}

Since the purpose of an antireflection coating is to maximize light transmission, total transmissivity of quartz wafers with various coatings has been measured using a JASCO V-570 spectrophotometer. The coating was done on one side of the quartz wafer. ${ }^{19}$ An integrating sphere was used in the measurement, which collects transmitted light through a sample from all directions. Total transmissivity measurements at different incident angles have also been performed using a home-built monochromator-based spectroscopic setup with an integrating sphere. The white light from a

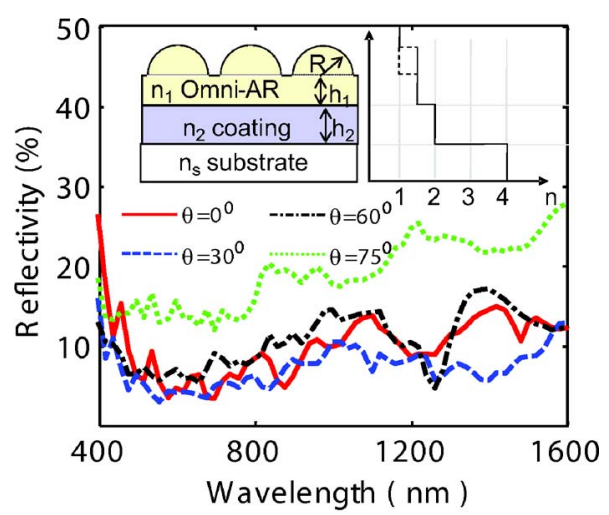

(a)

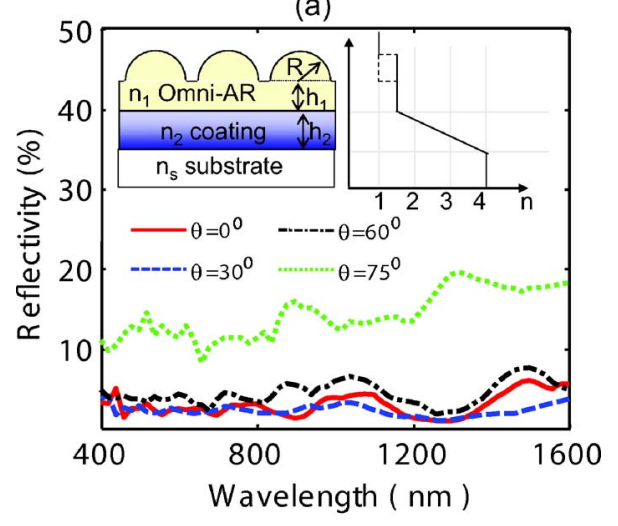

(b)

FIG. 10. (Color online) Reflectivity simulation results for index matching of silica omni-AR to $\mathrm{Si}$ substrate: (a) $\mathrm{SiN}_{x}$ film with a fixed index of 2.0 and (b) graded index film with index varying linearly from omni-AR $(n=1.5)$ to substrate $(n=4.0)$.

100-W Oriel quartz tungsten halogen lamp was focused on the sample at different incident angles through a flexible liquid light guide. Care was taken to ensure that the transmitted light through the sample is coupled into the integrating sphere. Due to limitations in the setup, the maximum incident angle is limited to $\sim 30^{\circ}$.

Figure 12 shows a normal-incidence total transmissivity measurement of quartz with an omni-AR coating, which comprises a monolayer of $2-\mu \mathrm{m}$ spherical silica particles immersed in a SOG film of $0.2-\mu \mathrm{m}$ thick. For comparison, the total transmissivity of a quartz wafer without any coating, a quartz wafer coated with $0.2-\mu \mathrm{m}$ SOG only and a quartz wafer coated with a monolayer of $2-\mu \mathrm{m}$ spherical silica particles only were also measured. The omni-AR coating improves the transmissivity from $\sim 88 \%$ to $\sim 92 \%$ at $400 \mathrm{~nm}$ and from $\sim 90 \%$ to $\sim 92 \%$ at $1100 \mathrm{~nm}$, demonstrating its broad-spectrum effect. Since the bare quartz wafer already has a high transmissivity above $88 \%$, the improvement by the omni-AR coating is tainted by the high background transmissivity. A $0.2-\mu \mathrm{m}$ SOG film alone slightly improves the transmissivity at short wavelengths by $\sim 1 \%$, possibly due to its smaller refractive index $(\sim 1.39)$, than that of quartz $(\sim 1.55)$. The reduced surface roughness due to SOG could be another contributing factor here. A monolayer of $2-\mu \mathrm{m}$ silica spherical particles alone decreases the transmissivity by $\sim 1 \%$ in the entire spectral range of interest. This is likely due to reflection from the multiple surfaces in the monolayer particle coating: the top surface of the particles, the bottom 


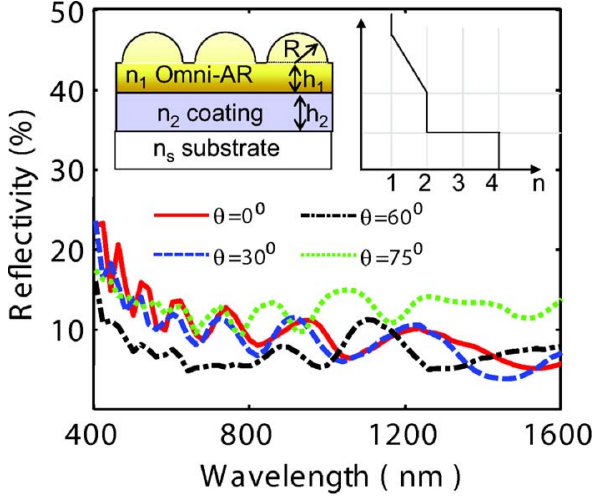

(a)

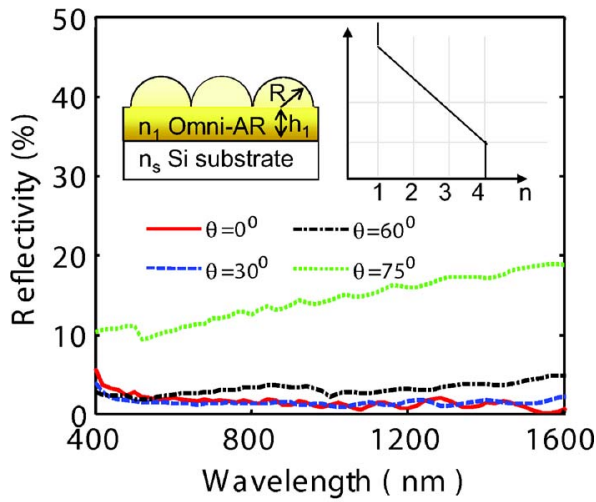

(b)

FIG. 11. (Color online) Reflectivity simulation results for graded index omni-AR structures on high index $\mathrm{Si}$ substrate: (a) index profile from air $(n=1.0)$ to $\mathrm{SiN}_{x}$ film below $(n=2.0)$ and (b) ideal index matching from air to Si substrate ( $n$ varying from air $n=1.0$ to Si $n=4.0$ ).

surfaces of the particles, and the surface of the quartz wafer. Light is scattered away from the multiple surface of these particles. Therefore, the SOG film not only serves as a glue to hold together the coating, but also reduces the number of internal surfaces which cause reflection.

An incident-angle dependent transmissivity measurement for an omni-AR coating on a quartz wafer, along with the schematic experimental setup, is shown in Fig. 13. Within the limited range of incident angle $\left(\sim 30^{\circ}\right)$, the omni-AR coating improves the total transmissivity from $\sim 87 \%$ to $\sim 92 \%$ at $500 \mathrm{~nm}$ and from $\sim 89 \%$ to $\sim 92 \%$ at $1000 \mathrm{~nm}$ for an incident angle of $30^{\circ}$. Under surface normal incidence, the measured transmissivity shows a similar spec-

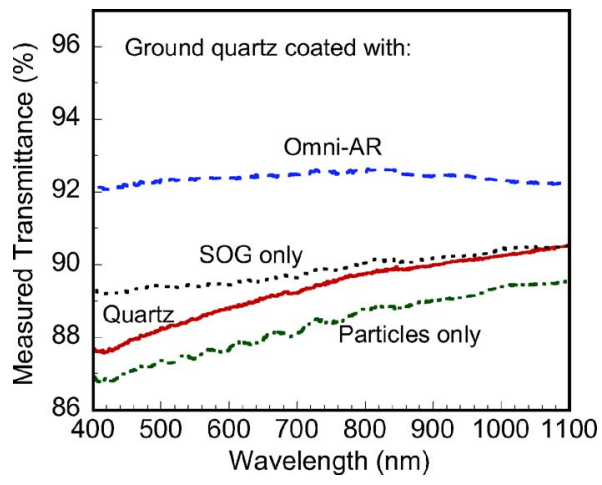

FIG. 12. (Color online) Total transmissivity under normal incidence of quartz wafers with various surface coatings.

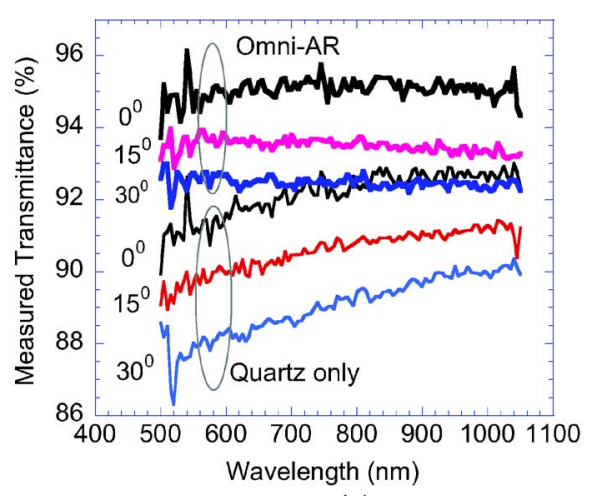

(a)

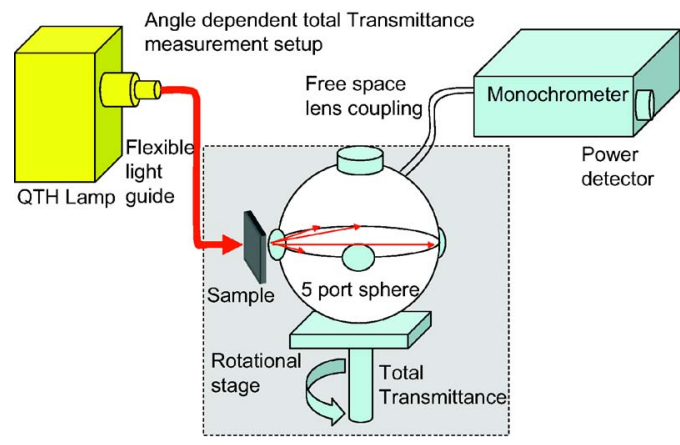

(b)

FIG. 13. (Color online) (a) Angle-dependent transmissivity of a quartz wafer before and after an omni-AR coating and (b) schematic of experimental setup for angle dependent total transmissivity measurement.

tral dependence for quartz and quartz with an omni-AR coating. However, the absolute transmissivity values are different. It is mostly due to the different experimental setups and variations in calibration.

Figure 14 is simulated transmissivity of quartz wafers with and without an omni-AR coating at different incident angles. To match the experimental conditions, the coating in the simulation consists of a $0.2-\mu \mathrm{m}$ SOG film with $2-\mu \mathrm{m}$ hemispherical particles on top (particle radius $R=1 \mu \mathrm{m}$ ). The wavelength-dependent refractive index is assumed to be that of silica, as shown in Fig. 3(b). However, the simulation does not take into account the reflection from the back surface of the quartz wafer, which could lead to slight reduction in transmission. The transmissivity increases from $\sim 95 \%$ to $\sim 98 \%$ with the coating at small incident angles of $0^{\circ}$ and

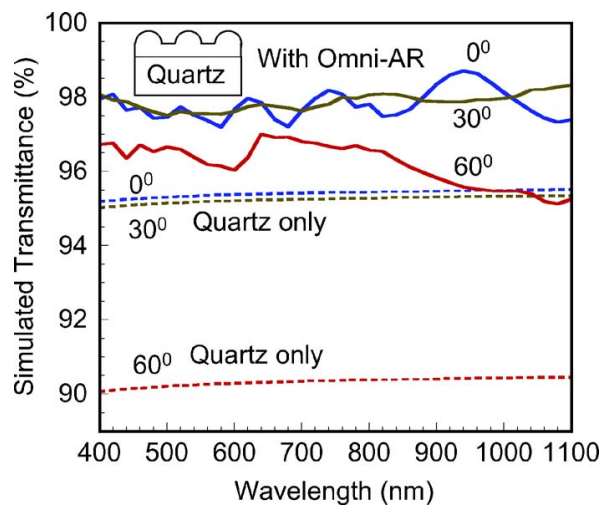

FIG. 14. (Color online) Simulated transmissivity at different incident angles for quartz wafers with and without an omni-AR coating. 
$30^{\circ}$. The results agree reasonably well with experiments in Figs. 12 and 13. The simulated results for large incident angles are also shown for completeness. With a large incident angle of $60^{\circ}$, the transmissivity increases from $\sim 90 \%$ to $\sim 96 \%$. Efforts are undertaken to build an experimental setup capable of measuring large incident angles.

\section{CONCLUSIONS}

A detailed analysis was presented to understand the reflectivity of microstructured surfaces for solar cell AR coatings. It was found that omnidirectionality (incident angle independent) antireflection can be achieved in various microstructured surfaces. Coupled with index matching to the substrate, close to zero reflectivity can be achieved on $\mathrm{Si}$ substrates with silica omni-AR coatings. Experimental results agree reasonably well with the theory. The results suggest that the proposed omni-AR structure is a promising and cost effective solution for current and future generation solar cells.

\section{ACKNOWLEDGMENTS}

The authors would like to thank Mr. K. Han, G. Song, and Z. Qiang for their help with transmissivity measurements. The work was supported by the Air Force Office of Scientific Research and National Science Foundation.

${ }^{1}$ A. Luque and S. Hegedus, Handbook of Photovoltaic Science and Engi- neering (Wiley, New York, 2003).

${ }^{2}$ J. A. Dobrowolski, D. Poitras, P. Ma, H. Vakil, and M. Acree, Appl. Opt. 41, 3075 (2002)

${ }^{3}$ D. Poitras and J. A. Dobrowolski, Appl. Opt. 43, 1286 (2004).

${ }^{4}$ J. A. Dobrowolski, Y. Guo, T. Tiwald, P. Ma, and D. Poitras, Appl. Opt. 45, 1555 (2006).

${ }^{5}$ A. Yariv and P. Yeh, Optical Waves in Crystals: Propagation and Control of Laser Radiation (Wiley-Interscience, New York, 2002).

${ }^{6}$ D. Bouhafs, A. Moussi, A. Chikouche, and J. M. Ruiz, Sol. Energy Mater. Sol. Cells 52, 79 (1998).

${ }^{7}$ W. H. Southwell, Opt. Lett. 8, 584 (1983).

${ }^{8}$ E. Yablonovitch, J. Opt. Soc. Am. 72, 899 (1982).

${ }^{9}$ P. Campbell and M. A. Green, J. Appl. Phys. 62, 243 (1987).

${ }^{10}$ M. C. Bautista and A. Morales, Sol. Energy Mater. Sol. Cells 80, 217 (2003).

${ }^{11}$ D. Lee, M. F. Rubner, and R. E. Cohen, Nano Lett. 6, 2305 (2006).

${ }^{12}$ D. Chen, Sol. Energy Mater. Sol. Cells 68, 313 (2001).

${ }^{13}$ J. Q. Xi, M. F. Schubert, J. K. Kim, E. F. Schubert, M. Chen, S. Y. Lin, W. Liu, and J. A. Smart, Nat. Photonics 1, 176 (2007).

${ }^{14}$ J. Zhao and M. A. Green, IEEE Trans. Electron Devices 38, 1925 (1991).

${ }^{15}$ E. Yablonovitch and G. D. Cody, IEEE Trans. Electron Devices ED-29, 300 (1982)

${ }^{16}$ J. Zhao, A. Wang, P. Campbell, and M. A. Green, IEEE Trans. Electron Devices 46, 1495 (1999).

${ }^{17}$ K. R. McIntosh, M. J. Cudzinovic, D. D. Smith, W. P. Mulligan, and R. M. Swanson, 3rd World Conference of Photovoltaic Energy Conversion (WCPVSEC-3) (IEEE, Osaka, Japan, 2003).

${ }^{18} \mathrm{~W}$. Zhou and M. Tao, Surface Coatings from Solutions, U.S. Patent pending.

${ }^{19}$ M. Tao, W. Zhou, H. Yang, and L. Chen, Appl. Phys. Lett. 91, 081118 (2007).

${ }^{20}$ M. J. Weber, Handbook of Optical Materials (CRC Press, Cleveland, 2003).

${ }^{21}$ M. G. Moharam and T. K. Gaylord, J. Opt. Soc. Am. 71, 811 (1981).

${ }^{22}$ K. C. Johnson, Appl. Phys. A: Mater. Sci. Process. 24, 249 (1981). 